\title{
DENTAL CARE FOR PREGNANT PATIENTS: A REAPPRAISAL
}

\section{WL Chai, WC Ngeow. Dental Care for Pregnant Patients: A Reappraisal, Annals Dent Univ Malaya 1998; 5: 24-28}

\begin{abstract}
This paper revisits the prescription of drugs for pregnant patients and the dental and oral changes during pregnancy. The management of these patients is also outlined. Emphasis should be placed on prevention of dental problem during pregnancy.
\end{abstract}

Keywords: dental care, pregnancy

\section{INTRODUCTION}

Pregnant patients are seen rather commonly in the dental offices. However, the possible side effects of dental care on these patients can be misunderstood by both the patients and dentists concerned. The dental management of pregnant patients is different from that of the routine dental patients because of associated physiological and hormonal changes in their body system. Because of these changes too, pregnant women need special dental care and treatment in order to minimise possible risk to the foetus and mother.

This paper revisits the prescription of drugs and the dental and oral changes during pregnancy. The management of pregnant patients is also outlined. The dental practitioners are strongly advised to consult the dental specialists, obstetricians and/or physicians in order to achieve the best management for the patients under their care.

\section{PRESCRIPTION OF DRUGS DURING PREGNANCY}

Although drugs administered to a pregnant patient have the mother as the principle target, the foetus invariably is also a recipient. Below is a review of the commonly used drugs in dentistry and their significance to the mother and foetus. This is meant to be a guideline and the dentist is advised to refer to the latest Dental Practitioners' Formulary, the British National Formulary or Brigg's Drugs in Pregnancy and Lactation for further details and recent advances.

\section{Antibiotics}

Some oral infections require antibiotic treatment. The benefit gained should be weighed before prescribing to pregnant patients as some of the drugs may cross the placenta and may have detrimental effects on the foetus.

As a general rule of thumb, it is preferred not to prescribe any unnecessary antibiotics to the pregnant patients. If the source of infection could be eradicated by other means, for example via endodontics, then this must the preferred options. Of course the endodontics treatment will be of palliative form as radiography is not advisable unless deemed urgent.

In choosing a suitable antibiotic, the dentist has to know what is its mode of action. Antibiotics like the Penicillin group act against the formation of bacterial cell wall, a fea-

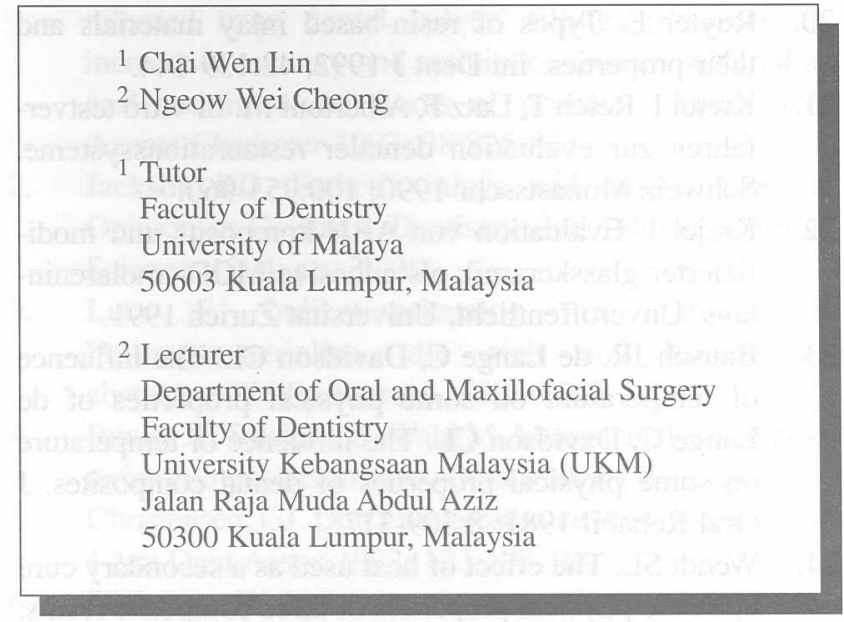

ture lacking in the human cells, thus rendering it a save drugs on the foetus.

Penicillin is the commonly prescribed antibiotics in the dental office because of its specificity and sensitivity against oral microbes. It passes the placenta but without causing records of hazardous effects on foetus (1). It has no detrimental effects to the human foetal cells as it acts by inhibiting the biosynthesis of a proteoglycan required for cell-wall synthesis, leading to bacterial lysis (2). It is the drug of choice for the pregnant patients, unless the patient has a history of allergy to Penicillin.

Erythromycin is usually prescribed as an alternative to Penicillin. It is a bacteriostatic drug but can become bactericidal in high dosages. It does not cause any teratogenic or toxic effect except for the estolate salt which may give rise to hepatotoxicity $(3,4,5)$. Therefore, Erythromycin estolate should bc avoided during pregnancy.

Clindamycin, a drug nowadays used as substitute for Penicillin allergy has not been linked to any known congenital defects (6). It can be used as an prophylactic antibiotic in pregnant patients with chronic rheumatic heart disease prior to dental treatment.

The use of cephalosporins during pregnancy is not contraindicated. There are no known reports of teratogenicity. The action of this class of antibiotics is directed toward preventing synthesis of the bacterial cell wall (1), just like that of the Penicillins.

Tetracyclines has been contraindicated for pregnant women and children under 12 years of age. They are associated with a significant risk for the development of hepatic or pancreatic injury (or both) in the pregnant patients (7). The most common complication in dentistry is discoloration of th dentition. It also poses the dentition to a hypoplastic matrix which result in an increased risk for dental caries. Tetracycline is also found to form chelates with calcium and this chelate can be deposited in th skeleton of the foetus, resulting in depression of the bone growth (4).

Metronidazole is a drugs usually prescribed to encounter anaerobic microbes. It is usual prescribed for dental abscess 
and as an adjunct to periodontal treatment. Metronidazole has been associated with an increased risk of midline facial defects (6). It has also been reported to be mutagenic in bacteria and carcinogenic in rodents (8). Thus, it is contraindicated in pregnant women (6). Other antibiotics that should be avoided during pregnancy include Vancomycin (Oto- and nephrotoxic) and Streptomycin (ototoxic)(9).

The control of orofacial infection is important in the pregnant women as bacteraemia, pyemia and septicemia are a threat to foetal health. Therefore, the dentist must justify the appropriate moment to start the proper antibiotics for these patients.

\section{Analgesics}

A patient in severe pain will have associated increase in the level of anxiety and an increase of smooth muscle contraction. This increase the risk of miscarriage. Therefore, pain control is very important during pregnancy. It is preferable to identify the cause of pain and eliminate it (10), for example by removing the offending tooth or treating it with root canal therapy rather than relying on symptomatic relief with analgesic medications. Such medication should be used as an adjunct to the procedures done. When analgesic is indicated, the dentist should try to control the pain with minimal dosage and potency for the shortest possible duration $(2,10)$. The potential benefit to the patient must outweigh the potential harm to the foetus (2).

Acetaminophen or commonly called paracetamol is safe to be used during pregnancy in therapeutic dosages. For the past 30 years, there have not been any reports of adverse maternal or foetal effects from the use of this drug during pregnancy (11).

The use of aspirin during pregnancy is controversial (6). Several studies have found no increase in birth defects with the use of salicylates (12). On the other hand, there have been reports of increase of incidence of oral clefts $(5,13)$, intrauterine death, growth retardation and pulmonary hypertension (6) with the use of aspirin/salicylates during pregnancy. Fetal intracranial haemorrhage has also been associated with the use of aspirin near term (5). It is suggested that its occasional usage during pregnancy is not harmful but its chronic use should be avoided (14).

Non-steroidal anti-inflammatory drugs work via inhibiting the cycloxygenase pathway. This inhibit the prostaglandin synthetase and ultimately may lead to premature closure of the ductus arteriosus (9). This may eventually leads to pulmonary hypertension with an increase of foetal mortality. Ibuprofen, a non-steroidal anti-inflammatory drug has been shown to have various and profound detrimental effects on foetal and neonatal circulation (15). For this reason, it is not advisable for pregnant patients to take drugs that inhibit the synthesis of prostaglandins.

The use of Codeine during pregnancy has been associated with cleft lip, cleft palate, cardiac defects, chest wall deformities, inguinal hernias and circulatory anomalies (5). Excessive dosage of narcotic drugs produce depression of the foetal central nervous system (16) while their long term use cause addiction in the foetus (18). Fortunately, narcotic drugs are not commonly prescribed in the dental clinics.

\section{Anaesthetics and sedative agents}

Pregnant women who are under a lot of pain or stress may have increased level of adrenaline (epinephrine). This emotional upset may be detrimental to the foetus. Therefore, pain control is fundamental for pregnant women undergoing dental treatment.

Local anaesthetic injection used in dental treatment like Lignocaine, Lidocaine and Prilocaine are safe to be used. They are not teratogenic and may be administered to pregnant patients in the usual clinical dosage (17). However giving large doses of Prilocaine are not advisable as this is known to cause methaemoglobinaemia, which could also cause maternal and foetal hypoxia (19). It is also considered safe practice to use a vasoconstrictor $(1: 100,000$ adrenaline/epinephrine) (20). Fellypressin is not advisable because of the theoretical risk on the uterus. It is a fallacy that pregnant patients shall not received any local anaesthesia as this drug only works locally or regionally and their systemic distribution to the foetus is very minimal indeed.

Bupivacaine and Mepivacaine are categorised in the Category C drugs in American Food and Drug Agency classification system (8). This means that either studies in animals have revealed adverse effects on the foetus (teratogenic or embryocidal, or other) or there are no controlled studies in women or studies in women and animals are not available. These drugs should only be given if the potential benefit justifies the potential risk to the foetus. In dentistry, this benefit can easily be achieved by using Lignocaine or Prilocaine. Thus, the use of Bupivacaine and Mepivacaine in pregnant patients is not advisable.

It is often advantageous to administer sedatives to allay a patient's anxiety associated with dental treatment. However, sedatives Benzodiazepines and Diazepam are contraindicated during pregnancy. They have been associated with birth defects, including cleft palate and lip $(8,18)$, cardiac defects and inguinal hernias $(5,21,22)$. Therefore, dental treatment or minor surgery under intravenous sedation is not advisable.

Barbiturates, an agent often used as an induction agent during general anaesthesia has bee' reported to cause anomalies in mice (5). All barbiturates can cross the placental membrane; thus they have potential adverse effect to the foetus. Chronic usage of barbiturate has been shown to cause withdrawal symptoms in postnatal infants (18) while removal of this drug from addictive mothers may cause premature labour and intrauterine foetal death (17). Fortunately this drug is not used in the dental clinics.

\section{DENTAL AND ORAL CHANGES DURING PREGNANCY}

The dental and oral changes during pregnancy can sometime be distressing. Pregnancy epulis and gingivitis are some of the common complaints a dentist may encounter in their practice. It is important that a good preventive programme is emphasised even prior to pregnancy so that the patients are aware of the problem they may encounter during their pregnancy. 


\section{Oral and dental changes during pregnancy Anaemia}

Pallor of the oral mucosa may be detected during pregnancy. This can be an indication that the mother is suffering from anaemia, most probably due to iron deficiency. This is a consequence of the increased iron requirement for the formation of the foetal red corpuscles. The iron requirement usually increases by $800 \mathrm{mg}$ during the pregnancy (2) and this can be met by giving the patient iron supplements. This is usually taken care of by the physician or obstetrician. The role of the dentist is more toward detecting this condition and making the appropriate referrals.

\section{Pregnancy epulis}

There is an increase in frequency of pregnancy epulis/ tumours in pregnant women, mostly during the second trimester (8). This lesion histologically mimicks a pyogenic granuloma (8). It represents an exaggerated inflammatory response to local irritation (2). It usually presents as a painless red spherical protruding swelling on the gingival margin or interproximal spaces and may bleed easily and is usually found on the canine-premolar region.

It is a fallacy that all these lesions must be excised surgically. Excision is only indicated when the mass interferes with chewing, brushing or oral hygiene procedures. Otherwise, they will normally subside after delivery.

\section{Pregnancy gingivitis}

Pregnancy gingivitis is frequently manifested during pregnancy even in those with good oral hygiene prior to pregnancy (23). It is a fallacy that they may have neglected their oral hygiene on the event of pregnancy. In fact, it has been suggested that this is due to hormonal changes associated with pregnancy, resulting in an increase of oestrogen and progesterone (24). This causes the gingiva to swell up (25, 26), rendering routine oral hygiene care more difficult. Other researchers had suggested that there is an accentuated response to dental plaque during pregnancy (2). Moreover, because of the presence of bleeding during toothbrushing, some patients may avoid the area altogether. So a cycle has been formed. In order to break this cycle, the patient must be reassured and given proper maintenance.

\section{Teeth mobility}

In addition to the gingival changes, generalised tooth mobility especially in the third trimester can be observed (8). This is possibly caused by some qualitative changes in the fibrous portion of the periodontal ligament (29). It is a fallacy that this will contribute to severe periodontal problem. Again, the patients should be reassured that this condition will settle after delivery. They should be encouraged to maintain a good oral hygiene in order to prevent periodontitis from settling in, as this is the actual cause for severe periodontal problem in the future.

\section{Caries}

The increase in incidence of caries during or following pregnancy may be due to the following reasons: i) gingival tenderness obscured the patient to maintain a good plaque control which ultimately cause increase caries formation (8)

ii) gastric acids associated with morning sickness may lead to enamel demineralisation (8)

iii) mothers are often advised to eat smaller amount of carbohydrate containing food more often due to the decreased of stomach capacity. This would lead to the more frequent exposure to acid challenges (8)

iv) some pregnant women have a craving for specific foods. These may be sour foods which contain a high level of acid or other foods which is sticky and have a high concentration of sucrose.

Oral hygiene procedures and frequent water rinses will help prevent decalcification due to morning sickness or exposure to lots of acidic foods (2). If vomiting continues, the daily topical application of fluoride gels will help reduce decalcification. Flexible mouthguards can be fabricated for the application of neutral sodium or stannous fluoride gels (2).

\section{TREATMENT PLANNING}

Treatment planning begins with appropriate triage. Emergency procedures, such as those engendered by serious trauma or infection, should be performed at any time with careful planning to minimise adverse effects (6). Urgent procedures that can be delayed are best performed after the first trimester. Completely elective procedures are best postponed until six weeks postpartum, which allows maternal physiology to return to the prepregnant state (27).

\section{Trimester Approach}

Studies suggest that the ideal dental treatment should include two visits during the first trimester, at least once during the second trimester and once during the third trimester (10). This is called the trimester approach.

The first visit should include a clinical examination coupled with most recently available radiographs. Areas that could possibly pose a problem or discomfort before delivery should be scheduled for restoration during the second trimester. Emergency treatment, updating history and medications can also be done at the initial visit of every case. The initial visit should also include prevention counselling. The patient should be educated of the more common dental problems that occur during pregnancy and the appropriate approach taken to prevent or treat these problems. Information on postnatal dental care and fluoride supplements can also be provided at this visit.

The second visit during the first trimester is needed to check the patient's compliance of home oral hygiene care, to observe hormone-related tissue changes and to reinforce prevention instructions.

The second trimester is the ideal time to perform dental treatment. The patient is usually more comfortable and is still able to recline in the dental chair. During this time, dental treatment proceed as for any other patients, but precautions noted previously should be observed.

A preventive oral care may be scheduled in the early 
third trimester. Appointments for these patients should be kept as brief as possible.

\section{Reinforcement}

For the pregnant patients who require no dental treatment during the second trimester, a single visit is sufficient. Patients who have no dental disease or home care deficiencies at this appointment may be excused from the third trimester appointment (10). Postnatal dental care for the newborn may be emphasised at this time. Patients who have persistent tissue inflammation, plaque and advancing caries during the first six months of pregnancy should be seen again in the third trimester. Patients with mild to moderate gingivitis should have another prophylaxis and another oral hygiene instruction.

\section{RADIOGRAPHY}

Although it has been known that the dental diagnostic radiograph causes minimal radiation to the foetus, it is suggested that radiographs should be avoided during pregnancy $(2,28)$. Usually the most recent films available in the patient's dental chart will be sufficient during the nine months of pregnancy (10).

It is recommended that radiographs only be taken for emergency care during the first trimester (2). When radiographs are essential, a properly shielded and collimated beam and high speed film are recommended. Proper collimation and shielding of the equipment restrict the size of the primary beam and reduce scatter radiation. The patient's abdomen needs to be shielded with a lead apron.

\section{PREVENTIVE MEASUREMENT}

\section{Plaque control}

Patients who are reluctant to floss must understand the added importance of interproximal plaque control. Those who would never think of using plaque-disclosing methods should be encouraged to do so at least once a week. Patients should be advised to brush after each snack that contains sugar. Also, some form of oral ravage, rinsing or brushing is imperative after regurgitation (30). The pregnant patient should be educated that through the efforts of increased plaque control alone, most dental diseases associated with pregnancy like gingivitis and caries can be avoided (31).

Some patients may avoid dental treatment altogether during pregnancy out of fear that it may harm the foetus and because of social belief. For the patient receiving regular dental care, this brief absence from the dental office is not a problem. However, to avoid necessary treatment out of ignorance or fear is unwise. The possible harmful results of dental neglect, like severe episode of pericoronitis or abscess can far outweigh any risks associated with dental treatment. Proper patient education is the key to break this belief.

\section{Nutritional support}

Pregnant patients normally receive nutritional guidance from their obstetricians. The dental team should be prepared to reinforce these informations. Nutrition and diet are important areas of preventive dentistry for the mother and foetus (10).
Nutritional deficiencies can lead to hypoplastic enamel in the primary incisors (32). There are nutritional guidelines that document ideal levels of all nutrients before, during and after pregnancy and the patients should be advised to refer to them.

It is a fallacy that the mother "loses a tooth for every child", a notion which stems from the mistaken belief that dental caries occurs because of the loss of calcium from the teeth to the growing foetus during pregnancy. Studies have shown that calcium is bound to the tooth structure and it cannot be reabsorbed back to the circulation like that of the bony skeleton. The pregnant patients should be made aware of this fact and not to blame pregnancy for their dental caries.

It is imperative that oral care should be advocated as early as possible during pregnancy to minimise the incidence of caries. This advice should be periodically reinforced.

\section{Fluoride supplementation}

The value of prenatal fluoride supplementation to prevent future caries in the child has been controversial and not been confirmed. Neither the American Academy of Pediatrics nor the American Academy of Pediatric Dentistry recommends prenatal supplementation (8)

Some study have shown that teeth morphology would have relatively flat occlusal surfaces with no deep pits or open fissures if prenatal fluoride supplement was given (33). Despite this finding, Thylstrup concluded that there was no biologic rationale for prenatal fluoride administration (33).

At the moment, there is still no adequate information regarding the efficacy of prenatal fluoride supplementation. There is suggestion that the fluoride supplements are not needed by those who live in fluoridated communities (10) Others suggested that in fluoridated areas, the optimal dosage of fluoride supplements is of $1.0 \mathrm{mg}$ fluoride ion (2.2 ma) once daily on empty stomach from the third month of pregnancy to term (2). In non-fluoridated areas, 1.5 to $2.0 \mathrm{mg}$ daily is recommended (33).

\section{CONCLUSION}

When prescribing pregnant patients with drug(s), the potential benefits to the patient must outweigh the potential harm to the foetus. The dentist should prescribed commonly used medications with good proven tract records. Trial of new drug(s) should be avoided at all during pregnancy.

Elective dental treatment should be avoided during the first trimester. Ideally, prior to pregnancy the patient should be encouraged to maintain a good dental hygiene. Whenever necessary, emergency procedures can be performed any time during pregnancy and elective surgery scheduled during the second trimester.

Dental radiography is safe, but must only be done for emergency cases. Minor dentoalveolar surgery and periodontal therapy can be performed without difficulty during week 13 through 24 and later, depending on the comfort of the patient. However, consultation with the patient's physician and/or obstetrician is always appropriate.

Dentists should encourage all patients of childbearing age to seek oral health counselling and examination as soon as 
they learn they are pregnant. The role of fluoride supplement to the developing dentition in the foetus remains controversial.

\section{ACKNOWLEDGEMENT}

Grateful thanks to Professor Dato' Dr. Hashim b. Yaacob for his kind assistance in editing this paper.

\section{REFERENCES}

1. Schwarz RH. Considerations of antibiotic therapy during pregnancy. Obstet Gynecol 1981; 58: 9599S.

2. Rothwell BR, Gregory CEB, Sheller B. The pregnant patient: considerations in dental care. Spec Care Dent 1987; 7: 124-9.

3. Barron WM. Medical evaluation of the pregnant patient requiring nonobstetric surgery. Clin Perinatol 1985; 12: 481-96.

4. Weinstein AJ. Treatment of bacterial infections in pregnancy. Drugs 1979; 17: 56-65.

5. Briggs GG, Boden Dorfer TW, Freeman RK, Yaffe SJ. Drugs in pregnancy and lactation: a reference guide to fetal and neonatal risk. Baltimore: Williams \& Wilkins Co., 1983: 286-313.

6. Fiese R, Herzog S. Issues in dental and surgical management of the pregnant patient. Oral Surg Oral Med Oral Pathol 1988; 65: 292-7.

7. Witter FR, King TM, Blake DA. The effects of chronic gastrointestinal medication on the fetus and neonate. Obstet Gynecol 1981; 58: 79-84.

8. Miller MC. The Pregnant Dental Patient. Calif Dent Assoc Journal 1995; 23: 63-70.

9. Gilman AG, Goodman LS, Gilman A. Goodman and Gilman's pharmacological basis of therapeutics. 6th edn. New York: Macmillan Co, 1980.

10. Chiodo GT, Rosenstein DI. Dental treatment during Pregnancy: a preventive approach. J Am Dent Assoc 1985; 110: 365-8.

11. Collins E. Maternal and fetal effects of acetaminophen and salicylates in pregnancy. Obstet Gynecol 1981; 58: 57- 61.

12. Heinonen OP, Slone D, Shapiro S. Birth defects and drugs in pregnancy. Littleton, Massachusetts: Publishing Sciences Group, 1977: 286-313.

13. Corby DG. Aspirin in pregnancy: maternal and fetal effects. Pediatrics 1978; 62: 930-7.

14. Rudolph AM. The effects of nonsteroidal antiinflammatory compounds on fetal circulation and pulmonary function. Obstet Gynecol 1981; 58: 63-66.
15. Beck-Coon RJ, Beck-Coon KA. Dental treatment in the pregnant or nursing patient. Gen Dent 1982; 30; 237 40.

16. Hill RM, Stern L. Drugs in pregnancy: effects on the fetus and newborn. Drugs 1979; 17: 182-97.

17. Sexton J, Edelin K, Booth DF, Kilgore T. Surgery in a gravid patient. J Oral Surg 1978; 36: 878-86.

18. Davis AF, Moir DD: Anesthesia during pregnancy. Clinics in Anesthesiology 1986; 4: 233-45.

19. Rose L, Kaye D. Internal Medicine for Dentistry. 2nd edn. St. Louis: 1073-6, 1990.

20. Safra M J, Oakley GP Jr. Association between cleft lip with or without cleft palate and prenatal exposure to diazepam. Lancet 1975; 2: 478-80.

21. Saxen I, Saxen L. Letter: Association between maternal intake of diazepam and oral clefts. Lancet 1975; 2:498.

22. Gaudie WM. Influences on periodontal disease: 1. Nutritional and hormonal factors. J New Zealand Soc Perio 1988; 66: 7-14.

23. Hugoson A. Gingivitis in pregnant women. Odontol Rev 1970; 21: 1-20.

24. Poma PA, Zajdzinski CV, Rana N, Edward LC, Webster A, Septo RC. Oral cavity evaluation, a part of prenatal care. IMJ III Med J 1979; 155: 85-8.

25. Grant DA, Stern IB, Everett FG. Orban's periodontics, a concept-theory and practice. St. Louis. CV Mosby Co., 1972.

26. Loe H. Periodontal changes in pregnancy. J Periondotol 1965; 36: 37-44.

27. Blass NH. Nonobsteteric surgery in the pregnant patient. Am Soc Anesthesiology Rev 1984; 12: 25-31.

28. Little JW, Fallace DA. Dental management of the medically compromised patient. 4th edition, St. Louis: CV Mosby Co., 1993: 383-9.

29. Grier RE, James DR. Dental management of the pregnant patient. Dent Clin North Am 1983; 27: 419-28.

30. Tilk MA, Meister F. Treatment of periodontal disease associated with pregnancy. Dent Surv 1978; 54; 24-6.

31. Alfano MC (Guest Editor). Understanding the role of diet and nutrition in dental caries. Changing Perspectives in Nutrition and Caries Res. New York: Medcom Inc., 1979: 6.

32. Glenn FB, Glenn WD, Duncan RC. Prenatal fluoride tablet supplementation and improved molar occlusal morphology. J Dent Child 1984; 51: 19-23.

33. Thylstrup A. Is there a biological rationale for prenatal fluoride administration? J Dent Child 1981; 48; 123-5. 